\title{
A Modified Leaky-LMS Algorithm
}

\author{
Tajuddeen R. Gwadabe, Mohammad Shukri Salman, and Hasan Abuhilal
}

\begin{abstract}
The leaky least-mean-square (LLMS) algorithm was first proposed to mitigate the drifting problem of the leastmean-square (LMS) algorithm. Though the LLMS algorithm solves this problem, its performance is similar to that of the LMS algorithm. In this paper, we propose an improved version of the LLMS algorithm that brings better performance to the LLMS algorithm and similarly solves the problem of drifting in the LMS algorithm. This better performance is achieved at a negligible increase in the computational complexity. The performance of the proposed algorithm is compared to that of the conventional LLMS algorithm in a system identification and a noise cancellation settings in additive white and correlated, Gaussian and impulsive, noise environments.
\end{abstract}

Index Terms-Leaky least-mean-square, system identification, noise cancellation.

\section{INTRODUCTION}

The least-mean-square (LMS) algorithm [1] is one of the most famous adaptive filtering algorithms because of its simplicity and ease of analysis. This has made most researchers to improve the LMS algorithm and also to find solutions to some of its drawbacks. Some of these improved algorithms include: the normalized least-mean-square (NLMS) [2], variable step-size least-mean-square (VSSLMS) [3], etc. These improved algorithms generally improve the performance of the LMS algorithm in terms of convergence rate and mean-square-error (mse) value.

One of the main drawbacks of the LMS algorithm is the drifting problem as analyzed in [4]. This is a situation where the LMS algorithm generates unbounded parameter estimates for a bounded input sequence. This may drive the LMS weight update to diverge as a result of inadequate input sequence [4]. The drifting problem has been shown in [5]-[7] in details.

The leaky least-mean-square (LLMS) algorithm is one of the improved LMS-based algorithms that use a leakage factor to control the weight update of the LMS algorithm [5], [6]. This leakage factor solves the problem of drifting in the LMS algorithm by bounding the parameter estimate. It also improves the tracking capability of the algorithm, convergence and stability of the LMS algorithm.

One of the main drawbacks of the LLMS algorithm is its low convergence rate compared to the other improved LMSbased algorithms. In this paper, we propose a new algorithm that improves the convergence rate of the LLMS algorithm. This is achieved by employing the sum of exponentials of the error as the cost function; this cost function is a

Manuscript received December 1, 2013; revised February 18, 2014.

T. R. Gwadabe and M. S. Salman are with the Electrical and Electronic Engineering Department, Mevlana University, Konya, Turkey (e-mail: tgwadabe@yahoo.com, mssalman@mevlana.edu.tr).

H. Abuhilal is with the Higher Colleges of Technology for Men Abu Dhabi, UAE (e-mail: habuhilal@hct.ac.ae). generalized of the stochastic gradient algorithm as proposed by Boukis et al. [8]. A leakage factor is added to the sum of exponential cost function which makes the proposed algorithm a combination of the generalized of the mixednorm stochastic gradient algorithm with a leaky factor.

This paper is organized as follows. In Section II, a review of the LLMS is introduced. In Section III, the proposed algorithm is introduced. In Section IV, experimental results are presented and discussed. Finally, the conclusions are drawn.

\section{LEAKy LEASt MEAN SQUARE AlgORITHM}

In system identification, the output of a linear system with input $x(k)$ is given by;

$$
d(k)=\mathbf{h}^{T} \mathbf{x}(k)+v(k),
$$

where, $\boldsymbol{h}$ is the impulse response of the system, $\mathbf{x}(k)$ is the tap-input signal and $v(k)$ is an additive noise, $T$ is transposition operator. The cost function of the leaky-LMS is given by;

$$
J(k)=e^{2}(k)+\gamma \mathbf{w}^{T}(k) \mathbf{w}(k),
$$

where, $\mathbf{w}(k)$ is the filter-tap weight, $\gamma$ is the leakage factor $(0<\gamma<1)$ and $e(k)$ is the error defined by;

$$
e(k)=d(k)-\mathbf{w}^{T}(k) \mathbf{x}(k) .
$$

The filter-tap can be recursively updated by;

$$
\mathbf{w}(k+1)=(1-\mu \gamma) \mathbf{w}(k)+\mu \mathbf{x}(k) e(k),
$$

where, $\mu$ is the step-size that is defined by;

$$
0<\mu<\frac{2}{\gamma+\lambda_{\max }(\mathbf{R})},
$$

where $\lambda_{\max }$ is the maximum autocorrelation matrix of the input tap vector.

\section{Proposed Algorithm}

In order to improve the convergence rate of the LLMS algorithm, we propose a new algorithm that employs a sum of exponentials into the cost function of the LLMS algorithm gives a new cost function is defined as;

$$
J(k)=(\exp (e(k))+\exp (-e(k)))^{2}+\gamma \mathbf{w}^{T}(k) \mathbf{w}(k),
$$


where $e(k)$ is defined as in (3) above. Deriving (5) with respect to $\mathbf{w}(k)$, gives;

$\frac{\partial J(k)}{\partial \mathbf{w}(k)}=2(-\mathbf{x}(k) \exp (e(k))+\mathbf{x}(k) \exp (-e(k)))+2 \gamma \mathbf{w}(k)$

The tap-update is given by;

$$
\mathbf{w}(k+1)=\mathbf{w}(k)-\frac{\mu}{2} \frac{\partial J(k)}{\partial \mathbf{w}(k)},
$$

Substituting (6) in (7) and rearranging, the update becomes

$$
\mathbf{w}(k+1)=(1-\gamma \mu) \mathbf{w}(k)+2 \mu \mathbf{x}(k) \sinh (e(k)) .
$$

\section{Simulation Results}

The purpose of the experiments done in this section is to investigate the performances of the LLMS and the proposed algorithms in system identification and noise cancellation settings under different noise environments. All simulated results are obtained by 300 independent runs.

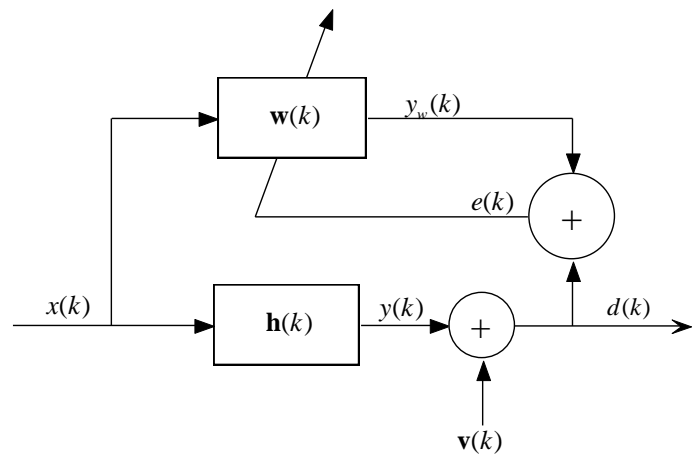

Fig. 1. System identification configuration.

\section{A. System Identification}

In this section, the system identification setting shown in Fig. 1 is used. The input signal is created using a first order autoregressive model (AR(1)) given by $x(k)=0.8 x(k-1)+\eta_{0}(k)$, where $\eta_{0}(k)$ is a white Gaussian process with zero mean and variance $\sigma_{\eta_{0}}^{2}=0.36$. The impulse response of the system is modeled by a low pass filter of 16 taps $(N=16)$ and a transfer function as shown in Fig. 2. The convergence rate and the mse are considered as measures. The simulations were done for stationary signal, corrupted with white and correlated Gaussian noise.

\section{1) Additive white Gaussian noise}

The signal in this experiment is assumed to be corrupted by an additive white Gaussian noise (AWGN) process with zero with and variance $\sigma_{v_{o}}^{2}=0.000225$. The simulations were done with a leakage factor, $\gamma=0.0001$ and $\mu=0.005$ for both algorithms. Fig. 3 shows the convergence of both algorithms with mse $=-39 \mathrm{~dB}$. The proposed algorithm converges at 1500 iterations while the conventional LLMS converges at 2500 iterations.

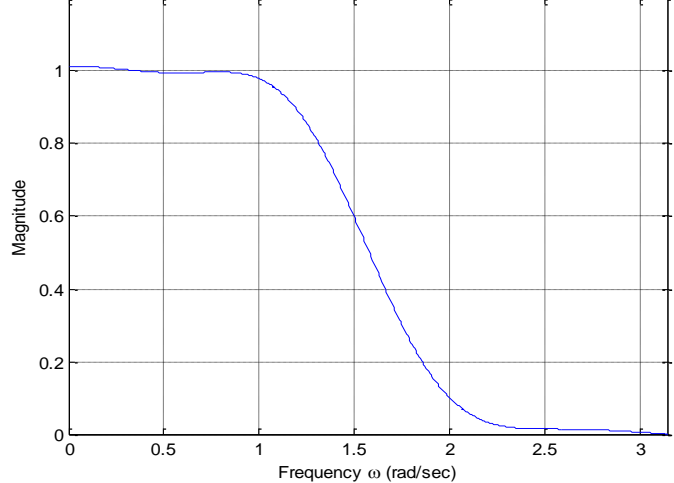

Fig. 2. Transfer function of the unknown system $\mathbf{h}(k)$.

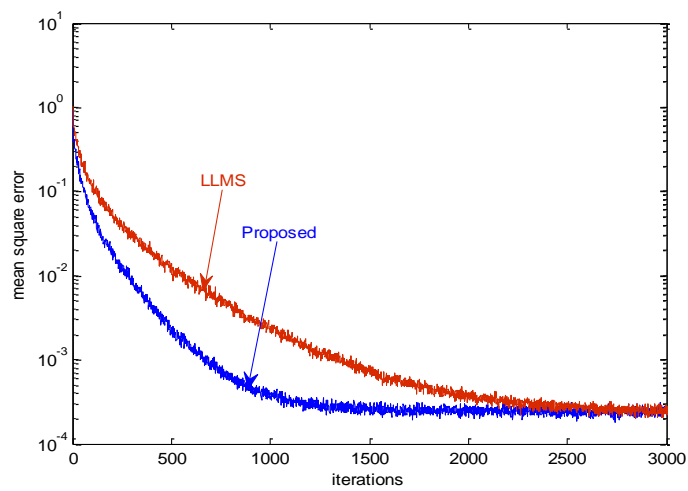

Fig. 3. The ensemble MSE for LLMS and the proposed algorithm in AWGN, $N=16, \gamma=0.0001$ and $\mu=0.005$ for both the algorithms.

\section{2) Additive correlated Gaussian noise}

In this experiment, the signal created in Section IV.A.a is assumed to be corrupted by an additive correlated Gaussian noise (ACGN) process. The ACGN is generated by $\mathrm{AR}(1)$ process, $v(k+1)=\rho v(k)+v_{0}(k)$, where $v_{0}(k)$ is a white Gaussian noise with zero mean and variance $\sigma_{v_{0}}^{2}=0.000225$, and $\rho$ is the correlation coefficient $(\rho=0.7)$. The simulations were done with the same parameter as the experiment in Section IV.A.a. Fig. 4 shows that the algorithms converge to the same mse $(\mathrm{mse}=-35 \mathrm{~dB})$. The proposed algorithm converges faster (1100 iterations) compared to the standard LLMSF (2100 iterations).

This shows that the modification done to the cost function of the LLMS algorithm improves the convergence rate of the LLMS algorithm both in white and correlated Gaussian noise environments in system identification setting.

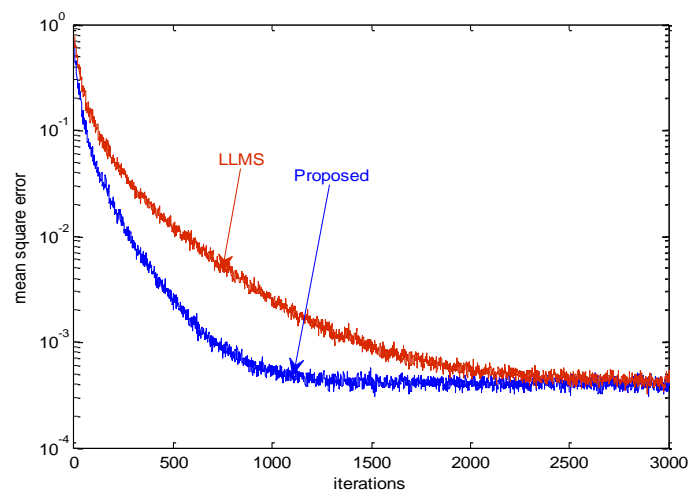

Fig. 4. The ensemble MSE for LLMS and the proposed algorithm in ACGN, $N=16, \gamma=0.0001$ and $\mu=0.005$ for both the algorithms. 


\section{B. Noise Cancellation}

In this section, a noise cancellation setting used to investigate the performance of the proposed algorithm as compared with that of the conventional LLMS is shown in Fig. 5. The input signal is assumed to be a Gaussian signal of zero mean and unity variance. A filter of length 32 taps is used and all simulations were done in white and correlated impulsive noise environments.

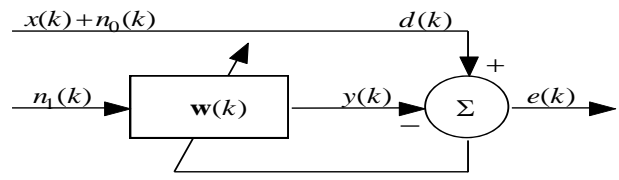

Fig. 5. Noise cancellation setting.

\section{1) Additive white impulsive noise}

Due to under water acoustic noises, man-made noise, atmospheric noises, etc., noise process is better to be modeled as impulsive rather than Gaussian noise [9], [10]. An impulsive noise can be generated using the probability density function: $f=(1-\varepsilon) G\left(0, \sigma_{v}^{2}\right)+\varepsilon G\left(0, \kappa \sigma_{v}^{2}\right)$, with variance $\sigma_{f}^{2}$, given by $\sigma_{f}^{2}=(1-\varepsilon) \sigma_{v}^{2}+\varepsilon \kappa \sigma_{v}^{2} \cdot G\left(0, \sigma_{v}^{2}\right)$ represents the nominal background noise with Gaussian distribution of zero mean and variance $\sigma_{v}^{2} \cdot G\left(0, \kappa \sigma_{v}^{2}\right)$ represents the impulsive part where $\kappa \geq 1$ and $\varepsilon$ are the strength and the probability of the impulsive components, respectively. In this experiment an additive white impulsive noise (AWIN) with zero mean and variance $\sigma_{v_{0}}^{2}=0.000225$ is used with $\kappa=100$ and $\varepsilon=0.2$. For both algorithms $\gamma=0.0001$ and $\mu=0.006$ were selected. Fig. 6 shows that the proposed algorithm converges faster than the LLMS by 250 iterations.

\section{2) Additive correlated impulsive noise}

In this experiment, an additive correlated impulsive noise (ACIN) is generated by using the $\mathrm{AR}(1)$ process given in Section IV, part A, title 2), where, $v_{0}(k)$ is a white impulsive noise process with variance $\sigma_{v_{0}}^{2}=0.000225$. The simulations were done using the same parameters in Section IV, part B, title 1). Fig. 7 shows that both algorithms converge to the same mse $(\mathrm{mse}=20 \mathrm{~dB})$, and the proposed algorithm converges faster than the conventional LLMS algorithm by 200 iterations.

As noticed from Fig. 6 and Fig. 7, the introduction of the sum of exponentials into the cost function of the LLMS algorithm, as in the proposed algorithm, significantly improves the convergence rate of the LLMS algorithm. This is shown by simulations in both AWIN and ACIN environments in noise cancellation setting.

\section{CONCLUSION}

In this paper, a new algorithm is introduced that improves the performance of LLMS algorithm by modifying its cost function. The performance of the proposed algorithm is compared to that of the conventional LLMS algorithm in system identification and noise cancellation settings. Simulation results show that the proposed algorithm outperforms the conventional LLMS algorithm in white and correlated, Gaussian and impulsive noise environments.

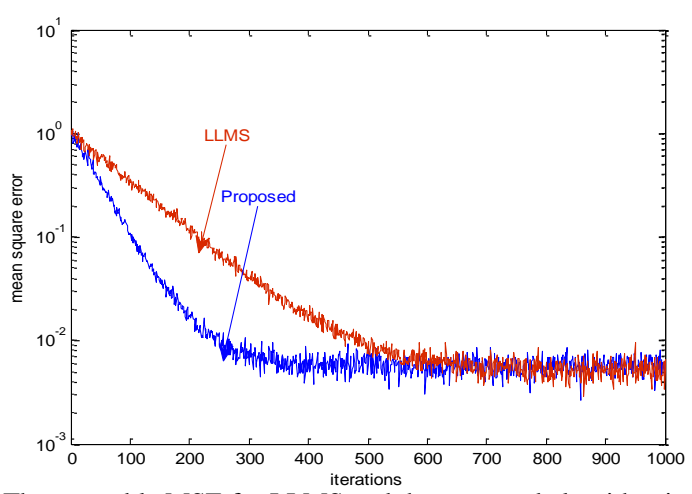

Fig. 6. The ensemble MSE for LLMS and the proposed algorithm in AWIN, $\mathrm{N}=32, \gamma=0.0001$ and $\mu=0.006$ for both the algorithms.

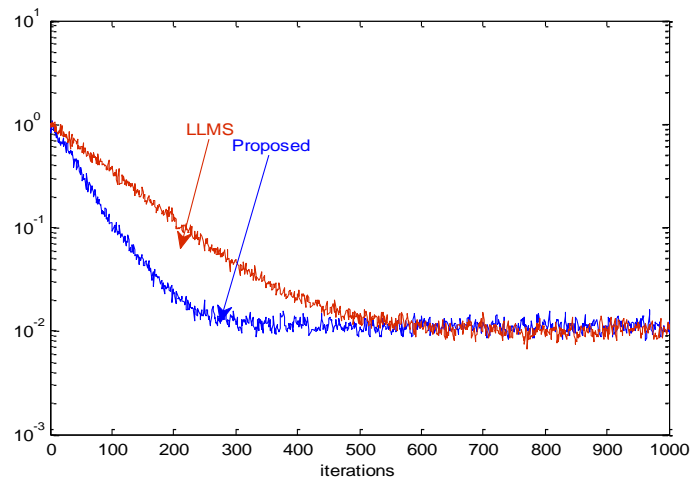

Fig. 7. The ensemble MSE for LLMS and the proposed algorithm in ACIN, $\mathrm{N}=32, \gamma=0.0001$ and $\mu=0.006$ for both the algorithms.

\section{REFERENCES}

[1] B. Widrow and S. D. Stearns, Adaptive Signal Processing, NJ: Prentice Hall, 1985.

[2] N. Bershad, "Analysis of then normalized LMS with Gaussian inputs," IEEE Transaction on Acoustic, Speech and Signal Processing, vol. 34, no. 4, pp. 793-806, 1986.

[3] R. Harris, D. M. Chabries, and F. Bishop, "A variable step (VS) adaptive filter algorithm," IEEE Transactions on Acoustic, Speech and Signal Processing, vol. 34, no. 2, pp. 309-316, 1986.

[4] W. A. Sethares, D. A. Lawrence, C. R. Johnson, and R. R. Bitmead, "Parameter drift in LMS adaptive filters," IEEE Transactions on Acoustic, Speech and Signal Processing, vol. 34, no. 4, pp. 868-879, 2003.

[5] V. H. Nascimento and A. H. Sayed, "Unbiased and stable leaky-based adaptive filters," IEEE Transactions on Signal Processing, vol. 47, no. 12, pp. 3261-3276, 1999.

[6] D. A. Cartes, L. R. Ray, and R. D. Collier, "Lyapunov tuning of the leaky LMS algorithm for single-source, single-point noise cancellation," in Proc. the American Control Conference, Alington, VA, 2001, vol. 5, pp. 3600-3605.

[7] K. A. Mayyas and T. Aboulnasr, "Leaky-LMS: a detailed analysis," in Proc. IEEE International Symposium on Circuits and Systems, 1995, vol. 2, pp. 1255-1258.

[8] C. Boukis, D. P. Mandic, and A. G. Constantinides, "A generalised mixed norm stochastic gradient algorithm," in Proc. $15^{\text {th }}$ International Conference on Digital Signal Processing, Cardiff, 2007, pp. 27-30.

[9] H. Delic and A. Hocanin, "Robust Detection in DS/CDMA," IEEE Transactions on Vehicular Technology, vol. 51, no. 1, January 2002.

[10] M. S. Ahmad, O. Kukrer, and A. Hocanin, "An efficient recursive inverse adaptive filtering algorithm for channel equalization," in Proc. European Wireless Conference, Lucca, Italy, 2010, pp. 88-92.

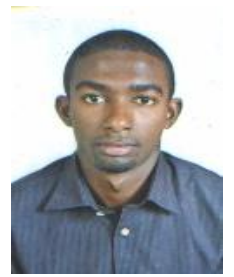

T. R. Gwadabe was born in 1987 in Nigeria. He received is B.Eng. in electrical engineering from Bayero University Kano (BUK), Nigeria, in 2011.

$\mathrm{He}$ is currently a master's student at Mevlana University, Turkey. His research interests include signal processing, adaptive filtering techniques, communications and image processing. 


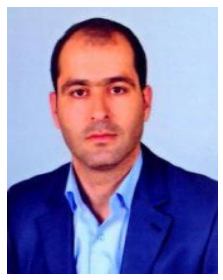

M. S. Salman was born in 1977 in Palestine. $\mathrm{He}$ received the B.Sc., M.Sc. and $\mathrm{PhD}$ degrees in electrical and electronic engineering from the Eastern Mediterranean University (EMU), in 2006, 2007 and 2011, respectively. From 2006 to 2010, he was a teaching assistant in Electrical and Electronic Engineering Department at EMU, in 2010; he joined the Department of Electrical and Electronic Engineering at European University of Lefke (EUL) as senior lecturer for the Department. Since 2011 he is working as an assistant professor in the Department of Electrical and Electronic Engineering at Mevlana (Rumi) University, Turkey. He served as a TPC and program chair for many international conferences. He is currently supervising $4 \mathrm{MS}$ and $4 \mathrm{PhD}$ theses. His research interests include signal processing, adaptive filters, image processing, sparse representation of signals, control systems and communications systems.

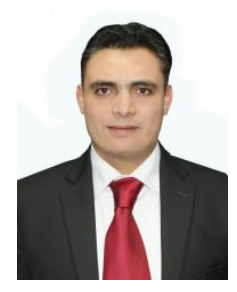

H. Abuhilal was born in Amman, Jordan in 1980. He received the B.Sc, M.Sc and $\mathrm{PhD}$ degrees in electrical and electronics engineering from Eastern Mediterranean University (Cyprus-Turkey), in 2002 2005 and 2012, respectively. From 2003 to 2007, he was a teaching assistant at Electrical and Electronics Engineering Department at EMU, in 2008; he moved to Mobile Systems International Company and served as radio frequency consultant engineer, for a mobile operator in the Kingdom of Saudi Arabia. In summer 2008 he joined the Math/IT Department at Dhofar University and served for 4 years, in 2013 he moved to a faculty position in the United Arab Of Emirates, at the Higher Colleges of Technology since then, he is working as instructor for the Electronics Engineering Department.

His research interests include Multi carrier communication, CDMA and multi input multi output communication, multi user detection, and $\mathrm{V}$ BLAST detection. 